\title{
A Case Study Evaluating the Impact of Human Behavior on a Manufacturing Process In-line with Automatic Processes by Means of a Simulation Model
}

\author{
E. Silva ${ }^{1}$, M. Donauer ${ }^{1}$, A. Azevedo ${ }^{1,2}$, P. Peças ${ }^{3}$ and E. Henriques ${ }^{3}$ \\ ${ }^{1}$ Faculty of Engineering of the University of Porto, Porto, Portugal \\ ${ }^{2}$ INESC TEC, Porto, Portugal \\ ${ }^{2}$ IDMEC, Instituto Superior Técnico, TULisbon, Lisbon, Portugal \\ (esilva@inescporto.pt)
}

\begin{abstract}
In many manufacturing systems human resources are essential in some cognitive intensive tasks while the more repetitive ones are assigned to automatic systems. If on the one hand, automation has a deterministic pace; humans are known by a flexible and variable work manner. Therefore, a reliable description of both hardware and human components is required for designing such manufacturing systems.

The purpose of this paper is to investigate the impact of the variable throughput of a manual process in a production flow that contains automatic processes upstream and downstream. With regard to the description of human behavior, two sources of variability were considered: natural and abnormal variability. Natural variability refers to the differences in terms of processing times that can be found among individuals. Organizational aspects such delays in shift changing and breaks along the shift, are referred as abnormal variability, and were also investigated by means of an analytical and simulation models.
\end{abstract}

Keywords - human performance, natural and abnormal variability, simulation model

\section{INTRODUCTION}

The recent market trends forced manufacturing companies to improve in terms of flexibility and reaction time to changes in market demands. This increased the needed level of process optimization. Therefore, highly automated systems assumed monotonous tasks, while operators retain the control of the most cognitive demanding ones. However, sometimes this increased responsibility and decision making roles placed the human operators in the weakest links in the operational loop [1]. In fact, it has been recognized by many authors that the performance of a human operator is time-varying and has a stochastic nature, which affect the stability of the manufacturing process [2]. Thus, the performance optimization that has been traditionally analyzed from the perspective of the machine performance progressively demands for models that aim at an optimization considering the human-machine interaction in the manufacturing systems. Because the actions of the human operators have a tremendous effect on the productivity of an industrial process and in many cases the paradigm of a fully automated factory is not advisable, the need for methods for quantifying operator performance and its variability become clear. There already exists a set of approaches and methods which consider human variability in the analysis of a manufacturing system. For instance, Mummolo [4] described a model that studies the effects of fatigue and learning phenomena on workers performance. Zülch [5] proposed a method to model other important human aspects such as individual abilities, talents, preferences and vocational restrictions. They use a personnel-oriented simulation tool Engpassorientierte Simulation von Personalstrukturen (ESPE) to optimize the planning and reassigning of personnel. Digiesi [6] proposed a detailed model for assembly lines consisting of a combination of highly automated workstations, workstations with a small degree of automation and manual processes. The variability in flow time was analyzed having trained workers and also in the presence of new workers, which significantly affect the line performance. The authors also analyzed the fatigue phenomenon and revealed there are some periods along the shift in which more products are more likely to be accumulated in the buffers. The final periods of the shift are generally more problematic.

In many production environments, skills and capabilities of workers cannot be replaced by automation. For this reason it becomes essential to develop simulation tools that account for a manual and automated component. In the early studies, these DES tools considered workers as resources, often neglecting their dynamic behavior. Still, most commercial simulation tools regard workers as simple resources, characterized either by a constant nominal performance or by a mathematical representation (normal distribution, triangular distribution, etc.) [6]. More recently there are models being proposed about the changing worker behavior over time and suggesting the use of DES as a personnel planning tool $[5,6]$.

This paper analyses the impact of some typical human behaviors and misbehaviors in a manufacturing production process. More specifically, the fact that humans may vary significantly in performance will be studied. Two kinds of variability were taken into account, defined as: natural variability and abnormal variability. Natural variability is considered to be the difference in processing time (pace) among different operators. Abnormal variability refers to the fluctuations in the availability of the workforce. The human resources unavailability due to daily breaks for coffee, lunch, etc. affect the flow in the processes where operators are directly involved. Even when breaks are formally scheduled and supposedly balanced, serious perturbations can occur. If other breaks or temporary absences are considered such as arriving late for shift, time for toilet and water, a stroll around to socialize, etc., the disturbances can increase significantly. The impact of 
those in the flow is relevant especially when the manual process is in line with automatic processes with an almost constant pace and a limited buffer in between.

Many companies routinely hit capacity constraints in some situations and immediately consider adding overtime for current workers or hiring workers for new shifts. This paper proposes a methodology for identifying bottleneck and over-capacity situations caused by the unstable throughput of a manual process. By combining the outputs of data analysis and simulation model, a diagnosis of the current situation of an industrial process is done. The results were generated by means of real input data obtained at the plant. Some suggestions may imply an adaptation of organizational and personnel structures aiming at an improved alignment between automatic and manual processes. We propose a more flexible personal structure that considers the possibility of re-organizing personal assignment along time.

The paper is organized as follows. A brief literature review of the related work is presented in Introduction. Section 2 clarifies the industrial case study while Section 3 analyses the industrial case under analytical and simulation tools. The last two sections are dedicated to results and possible improvement actions.

\section{INDUSTRIAL CASE UNDER STUDY}

In the following case a production flow consisting of three main steps is being analyzed according to its balance (Fig. 1). A fully manual process (MP) is embedded in two automated processes (AP1 and AP2) with a limited buffer capacity in between. The production rate of AP1, is determined by the product mix and maintenance schedule and can vary up to $16 \%$. MP step refers to a specialized manual process related to quality inspection. In the production process of this highly technical and safety product, quality inspection is essential at the end of the line and it is accomplished in two phases: MP and AP2. All products are inspected by a trained operator and only in case of being accepted; the product is released to AP2. Although production rate of AP1 is known and calculating the capacity, including a safety factor (considering an inspection time higher than the average time) for MP, is trivial, at certain periods along the shift, the buffer between AP1 and MP is either not sufficient or over-dimensioned. The asynchronous output from MP results in either a restraint to the production volume of AP1 or unutilized workforce at MP.

Preliminary observations at the manufacturing plant have shown that in addition to the natural variability of human performance, other sources of variability do also exist. In fact, organizational issues such as personnel arriving late to shifts, leaving prior to shift end and extending breaks, result in more variability, which, in the following, is referred to as abnormal variability.

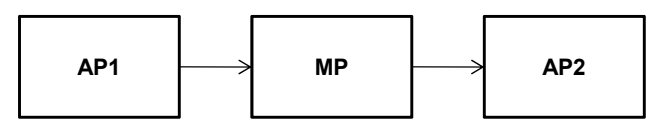

Fig. 1, Production process of the system under analysis.
The fact that MP has to co-exist with upstream and downstream automatic systems raises the challenge. Moreover, there is variability in the inspection times done by operators, and often the problem is how to match this variability with other processes.

One of the aspects addressed in this paper is that the time each operator takes to inspect a product changes significantly over time. For now the intention is only to quantify the variability in inspection times. For this reason data from 5 days (with 3 shifts each) was extracted from the databases containing the time spent to inspect each product. The total number of products in this sample is approximately 250,000 units. Fig. 2 illustrates the inspection times' distribution which has an average of 6.2 seconds per product. $87 \%$ of the products were inspected between 3 and 9 seconds but the boundaries of the distribution go further down to 1 seconds and maximum to 1 minute. The highest incidence of inspection times occurs between 4 and 7 seconds, which represents $68 \%$ of the sample. There were also products inspected in more than 1 minute in the system but after verifying with the operators, these intervals ( $>1$ minute) were assumed as being small breaks. Additionally there were products that were inspected in less than 1 second ( 2 in this sample) which was considered to be valid since they can be representative of a testing sample of a new product (which are marked differently and go without verification to the Development Department). The probability distribution that best fit the inspection time data is the Dagum or inverse Burr, widely used to describe income and wage distribution as well as wealth distribution [7]. A Dagum distribution function with three parameters $(k=1.5838$ $\alpha=3.9819 \beta=5.1861$ ) seems to be an adequate way to represent the variability in the inspection rate (according to EasyFit Standard(C version 5.5). Fig. 2 also includes a representation of the Log-normal distribution which will later be used in the simulation model and the normal distribution as a reference. The distribution of the inspection times was also acquired for each operator individually. MP runs with 24 operators at each shift and as mentioned before differences in performance among operators can be significant. As an example, while the global mean inspection time stands for 6.2 seconds, there are cases in which operators had a mean of 7.8 seconds while others of 5.4 seconds.

\section{MODELLING MANUAL INSPECTION}

\section{A. Understanding the inspection model by means of analytical methods}

Since MP is fed by an automated process with a deterministic throughput, one can think of the system as a tank with an inner and outer channel. While the diameter of the inner channel can be calculated (production rate of AP1), there is some uncertainty in determining the diameter of the outer channel (MP inspection rate). Even though, what goes into the tank must go out with limited room of accumulation for bottleneck situations. So, the system can assume three states: 


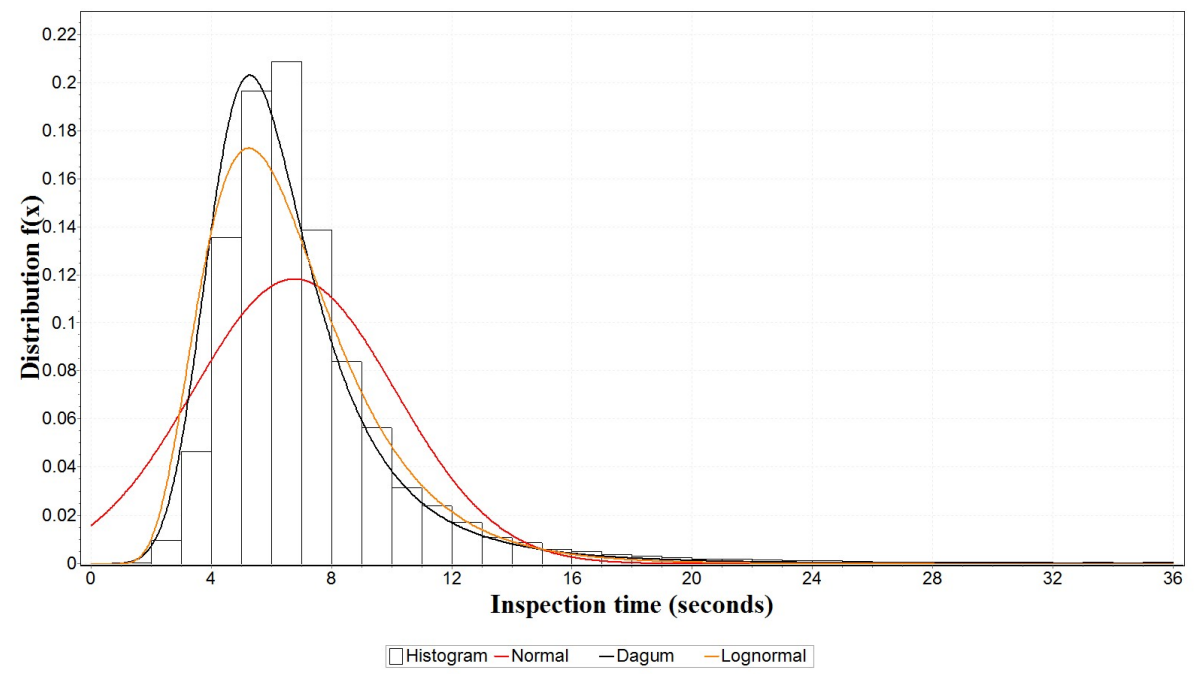

Fig. 2. Distribution of inspection times of MP.

$\Lambda<\mu$ Decreasing buffer between AP1 and MP, until no buffer is left;

$\Lambda=\mu$ Stable buffer;

$\Lambda>\mu$ Accumulating products in buffer over time until full;

For the scope of this study, $\Lambda$ is the mean production rate of AP1 and $\mu$ is the inspection rate in MP. The average production rate which can be treated as the average arrival rate at MP is 40 units $/ \mathrm{min}$. For matching this specific rate one needs to solve the following equation (1):

$$
\frac{1}{\Lambda}=\frac{1}{1 / \mu \times x}
$$

where $x=$ numbers of operators. The result is 4.13 operators, considering an average inspection time of 6.2 seconds/unit. However, a workforce of 6 operators is working all over the day, resulting in an estimated global inspection rate of $58 \mathrm{units} / \mathrm{min}$. If $\Lambda$ is significantly smaller than $\mu$ there must be excessive overcapacity. More detailed analytical calculations were done to understand why what seems to be an overcapacity situation sometimes results in buffer blockages upstream.

The operators are divided in 3 groups. When breaks are needed (for lunch and other smaller interruptions), the shift is organized in a way that only 1 group of operators is out. This means that in at certain periods of the day the capacity is reduced by one third. In periods in which 6 operators are working at MP the inspection rate is approximately 580 products inspected every 10 minutes, assuming an average inspection time of 6.2 seconds. On the other hand, in periods of reduced capacity (4 operators) the inspection rate decreases to 380 products inspected every 10 minutes. For simplification purposes, the production rate of AP1 is assumed to be almost invariable at a rate of 400 products being produced every 10 minutes. In periods in which there are 6 operators working, the exceeding capacity is $45 \%$, while in periods with reduced capacity there is a small difference between
AP1 and MP production rates. For a shift of 480 minutes, and accounting for all normal breaks, the percentage of periods with reduced capacity is $50 \%$, meaning that during 240 minutes, the number of operators available is 4. But considering that in half of the shift the exceeding capacity is $45 \%$ it is not understandable why there are periods in which the buffer is completely full and products have to be collected to a tank to avoid the blockage effect at AP1. One aspect to be considered is the concentration of the periods with reduced capacity. Taking a closer look at the shift model, one can identify a critical period. In the interval starting 180 minutes after the shift start until minute 360 (in the day shift this is equivalent to the period between $11 \mathrm{am}$ to $2 \mathrm{pm}$ ), the final inspection is performed with a reduced capacity (150 minutes out of 180 minutes). Additionally, two important factors are being neglected in this analytical model for simplification purposes, which can also contribute to buffer blockages. One is the relative disorder due to operator delays in shift change, the second is related to the natural variability of inspection time. These analytical calculations revealed that the nominal capacity installed exceed the needed capacity. In fact, the plant observations showed that there are moments along the shift in which the operators are idle waiting for products to arrive. But on the other hand, there are also moments in which the installed capacity is not enough to process all products and the buffer between AP1 and MP is completely full. Obviously, these analytical calculations are not representative of the reality. For this reason a simulation model was developed accounting for human variability, including the effect of natural and abnormal variability.

\section{B. Understanding the inspection model by means of discrete event simulation model}

Fig. 3 presents a flow chart of the simulation system and its variables. In this case the tool used to develop the DES model was Arena. A manual inspection process follows an automated production process with a buffer placed in between the two steps. 


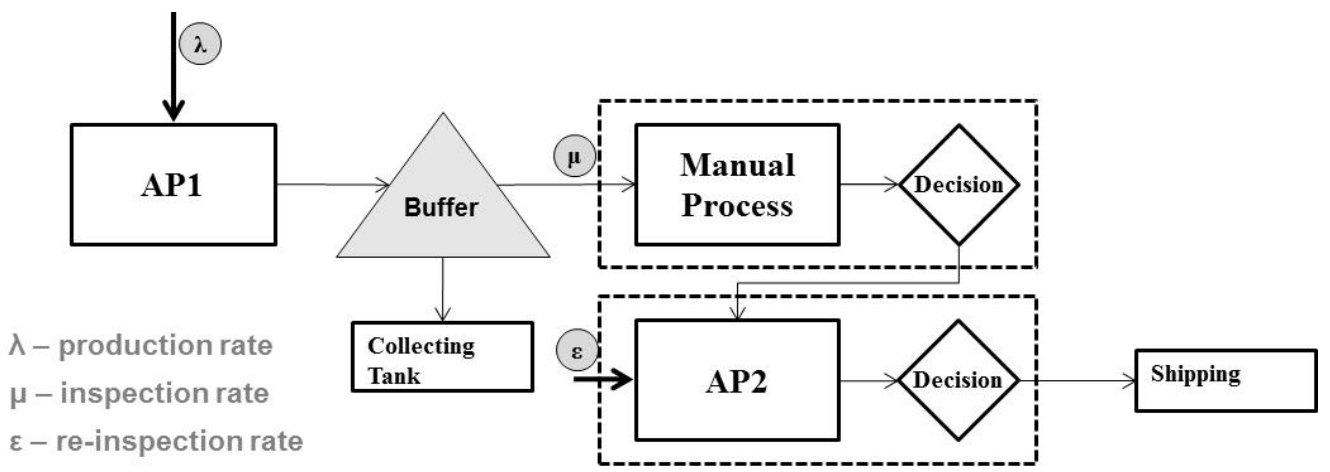

Fig. 3 Diagram of the process involved in the simulation model.

While in the real system AP1 can be stopped when the buffer is full, in the simulation model the products that could not enter the buffer were gathered at a collecting tank. In that way the impact of situations when the production is interrupted becomes quantifiable as the number of products that in the real system would not have been produced. An analysis of production data allowed for a quantification of inspection time with mean and standard deviation values. The manual inspection system is in detail modeled in the simulation together with conveyer speeds. Each of the 6 workstations in MP is operated by an operator who has his/her individual mean time and standard deviation taken from a historical database at the plant (modeled as a Log-normal distribution). In addition, each operator is following the real shift and break schedule. According to shift plans operators break for 10 minutes every hour and in addition 40 minutes for lunch. It is foreseen they are assigned in groups of 2 . Hence one can identify every hour a period of 30 minutes and during lunch a period of 180 minutes with a reduced service rate to 4 operators.

\section{RESULTS}

\section{A. SHIFT CHANGE ANALYSIS}

After having implemented the simulation model (Fig. 4 ), changes in the relevant variables can be done in order to identify the critical situations. Furthermore, the deep knowledge acquired during the observation periods at the plant and the analytical calculations provided indications of possible critical periods that should be carefully analyzed. The first aspect to be analyzed is the shift change. The fact that some operators leave prior to shift end and that some of the following operators arrive slightly (some minutes) late, create significant disturbances in the flow. As seen in the previous sections, there is exceeding capacity with full workforce and a buffer before MP to absorb some uncertainty regarding workforce and inspection time. The question to analyze is whether or not the current buffer and/or exceeding capacity is/are enough to deal with periods of transition between shifts.

To analyze in detail the effect of the shift change some variables were kept constant. The production rate and the individual inspection rates were not changed along the various simulations (40 units per minute for $\Lambda$ as in the analytical calculations and a statistical distribution assigned to each of the 6 operators which was neglected analytically). On the other hand, the time a station was without operator due to late arrivals and/or early leavings and the number of stations without operators was iteratively varied. Each time a variable was changed the simulation model was run with 10 replications.

Fig. 4 illustrates the evolution of the number of products leaving AP1 for the collecting tank (the buffer is full) for different configurations by varying the number of stations that were temporarily without an operator and their time of absence. From shop floor observations, situations in which only half of the workforce was in service were common. The results shown that for a constant production rate at AP1 and varying the time needed for the complete shift change to occur (moving from 6 operators to 0 and from 0 to 6 operators), there are some situations in which the buffer between AP1 and MP is not sufficient. In case 4 operators are absent for 5 minutes or more the buffer is not capable. When only 2 stations are idled, the shift change can go up to 20 minutes without blockages. These results tend to be consistent with the analytical calculations. As seen in the previous sections, the inspection rate with reduced workforce (4 operators) is not significantly lower that the arrival rate and if the period in which this occurs is not long the buffer should be able to absorb the variability. Even though the results point out the need to carefully control the way the shift change occurs because small variations in the workforce and time absence can originate blockages. Situations in which only half of the workforce is operating as observed in reality can only occur up to 9 minutes.

\section{B. LUNCH BREAK ANALYSIS}

To analyze the second critical moment (lunch break) a different set of simulations were run. In this case the simulations corresponded to the complete shift ( 8 hours), starting with 6 operators operating at the beginning of the shift and defining that after 180 minutes the number of operators was reduced to 4 according to the lunch schedules. The production rate at AP1 was varied for each simulation set. 


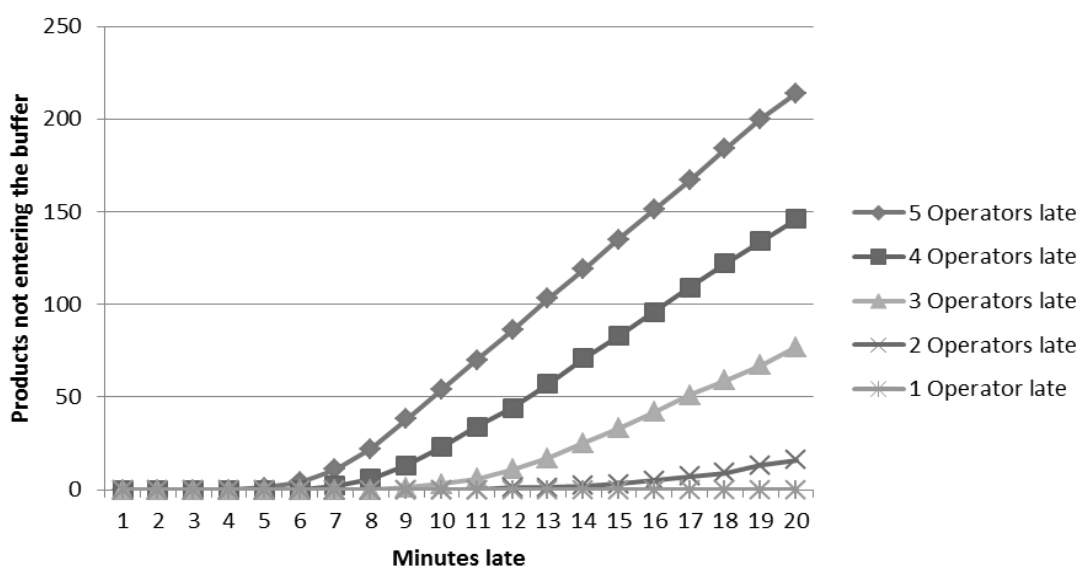

Fig. 4, Level of the buffer as a function of operators absent.

In accordance to plant observations, the simulation results show blockages in the lunch breaks for the current production rate 40 units per minute.

\section{POSSIBLE IMPROVEMENTS AND MAIN CONCLUSIONS}

The simulation model allowed for the understanding of the process flow and the impact of human variability in the throughput. The buffer that exists between AP1 and MP is of major importance to absorb a certain component of this variability. Even though, both industrial observations and simulation model results revealed that some blockages can occur, especially during shift change and lunch break. Expanding the buffer size is not desirable as a solution because increases in the production volume would again cause blockages. Another alternative would be to adjust the production rate according to the workforce availability (lower production rates in critical periods). Even though, other problems may be originated upstream in the process, since AP1 is the determinant pace of the complete manufacturing process. Instead, some actions should be done to contribute to a more stable output from MP. The first set of actions imply more strict rules regarding shift change to minimize the misbehavior of operators (leaving prior shift end and arriving late). A strategy to do so would be to adopt an hourly productivity bonus, instead of an overall daily productivity bonus. A daily bonus allows the operator to compensate lower production periods along the shift, while an hourly bonus would motivate a more stable output along the day. To minimize the negative impact of lunch breaks, one suggestion could be to have flexible operators trained to perform MP during lunch or other breaks. The simulation model was adapted to reflect this adjustment in the workforce. Several iterations were done, and the results show that if 5 operators were permanently at MP, there would be no blockages for a production rate of 40 products per minute. Actually the production rate can even increase to 45 products per minute and the process would still be stable. So, the proposal is to assign a set of 6 permanent operators to MP, divided in 3 groups. When one group makes a stoppage, one flexible and nondedicated operator assumes one position. This way, a permanent number of 5 operators are always guaranteed which softens the variability of MP performance along the day and minimizes the probability of blockages. Additionally if some operators of the permanent group could also be trained to other task, the effect of periods of overcapacity could also be reduced. Not only the production rate can increase, but also the utilization of the flexible operators for other tasks can occur.

\section{ACKNOWLEDGMENT}

This research is supported by $\mathrm{PhD}$ grants SFRH / BD / 33905 / 2009 and SFRH / BD / 33791 / 2009 of the Fundação para a Ciência e a Tecnologia (FCT). Furthermore, the support and cooperation of the managers and staff of the company, which remains to be anonymous, in the application case is highly appreciated.

\section{REFERENCES}

[1] R. Wang, J. Zhang, Y. Zhang and X. Wang: "Assessment of human operator functional state using a novel differential evolution optimization based adaptive fuzzy model", Biomedical Signal Processing and Control, vol. 7, pp. 490498, 2012.

[2] K. Tervo, L. Palmroth and H. Koivo: "Skill Evaluation of Human Operators in Partly Automated Mobile Working Machines", IEEE Transactions on Automation Science and Engineering, vol. 7, no. 1, pp. 133-142, 2010.

[3] H. Ovaskainen, J. Uusitalo and K. Vaatainen: "Characteristics and significance of a harvester operator's working technique in thinnings", International Journal of Forest Engineering, vol. 15, no. 2, pp. 67-77, 2004.

[4] G. Mummolo, G. Mossa, and S. Digiesi: "Learning and tiredness phenomena in manual operation performed in lean automated manufacturing systems: a reference model", International Intelligent Manufacturing Systems Forum, pp.341-346, 2004.

[5] G. Zulch, S. Rottinger and T. Vollstedt: "A simulation approach for planning and re-assigning of personnel in manufacturing", International Journal of Production Economics, vol. 90, pp. 265-277, 2004.

[6] S. Digiesi, A. Kock, G. Mummolo and J. Rooda: "The effect of dynamic worker behavior on flow line performance", International Journal of Production Economics, vol. 120, pp. 368-377, 2009.

[7] F. Domma and F. Perri: "Some developments on the logDagum distribution", Statistical Methods and Applications, vol. 18, no. 2, pp. 205-220, 2009. 